\title{
HAPS Wireless Communication Network Channel Modeling and Simulation
}

\author{
Xiao-Yang $\mathrm{Liu}^{1,2}$ \\ ${ }^{1}$ School of Computer Science and Engineering,Chongqing \\ University of Technology \\ Chongqing,400054, China \\ ${ }^{2}$ Postdoctoral Research Station of Information and \\ Communication Engineering \\ Chongqing University, Chongqing, 400030, China \\ 1xy3103@163.com
}

\section{Chao Liu}

School of Computer Science and Engineering,Chongqing University of Technology

Chongqing,400054, China

\author{
Wan-Ping Liu \\ School of Computer Science and Engineering,Chongqing \\ University of Technology \\ Chongqing,400054,China \\ Yi-Hao Zhang \\ School of Computer Science and Engineering,Chongqing \\ University of Technology \\ Chongqing,400054,China

\section{Xiao-Ping Zeng} \\ Postdoctoral Research Station of Information and \\ Communication Engineering, Chongqing University \\ Chongqing, 400030, China \\ zxp@cqu.edu.cn
}

\begin{abstract}
High altitude platform station (HAPS) is a new means of wireless communication method, which operate in stratosphere whose altitude is about $20 \mathrm{~km}$. In order to improve the channel quality of wireless communication transmission and signal-to-noise ratio.The wirless communication network of HAPS are studied in this paper. The performance of the wireless communication channel and channel model are analyzed and the characteristics of near space wireless channel are considered.The simulation results shows that signal level is different when the spacing distance is different.There is always a direct path between users and HAPS.
\end{abstract}

Keywords-high altitude platform station; near space; channel model; communication performance

\section{INTRODUCTION}

HAPS (High Altitude Platform Station) is an airship equipping radio transponders which stays in the stratosphere (the altitude of about $20 \mathrm{~km}$ ) whose weather conditions is comparatively stable for a long period of time. HAPS is a seamlessly connected information acquisition and distribution network formed by interconnected the near space vehicles, such as airship and unmanned aircraft. Present research is mainly focus on the near space vehicle development and design at home and abroad in order to offer the right bearing to near space network platform. The study of HAPS wireless communication network efficient network and transmission is relatively lagging. It mainly concentrated in the study of the multi-beam antenna design and cover scheme, and the capacity research of a single platform for the communication system, and so on [1-10].

The transmission rate, delivery reliability, and network lifetime are three fundamental but conflicting design objectives in HAPS networks. The references [11-17] propose a cross-layer analytical model for the study of network coding (NC)-based Automatic Repeat reQuest (ARQ) medium access control (MAC) protocols in correlated slow-faded (shadowed) environments, where two end nodes are assisted by a cluster of relays to exchange data packets.

The rest of this paper is organized as follows. In Section II, the HAPS wireless communication system model is set up. In Section III, the channel mathematical model is built up for the HAPS communication system. In Section IV, simulation analytical results are presented. Finally, conclusions are drawn in Section V.

\section{HAPS WIRELESS COMMUNICATION NETWORK}

The HAPS wireless communication network is drawn in Fig.1. HAPS network includes a set of forward can perform routing, and traffic management HAPS communication node. It can be implemented with a laser or microwave communication between them.It is a challenging problem about the link between HAPS [18-20].

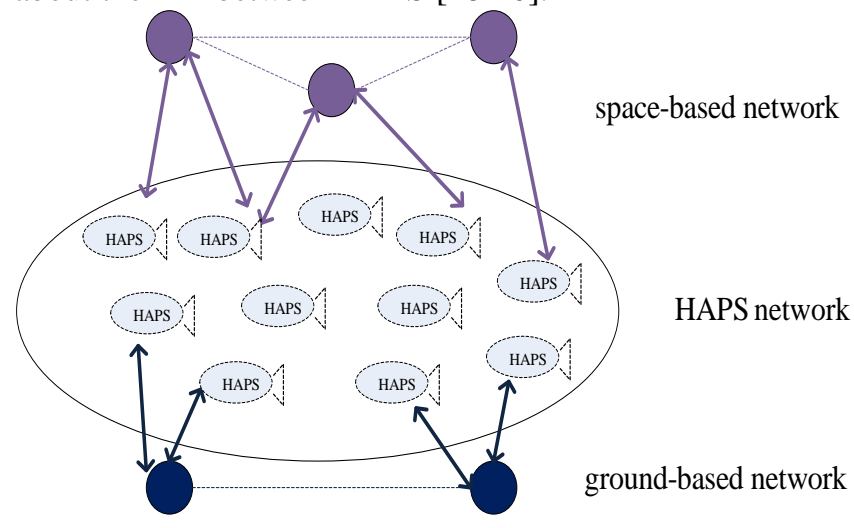


Figure 1. HAPS wireless communication network

The geometric relationships of a communications system of HAPS is shown in Fig.2.

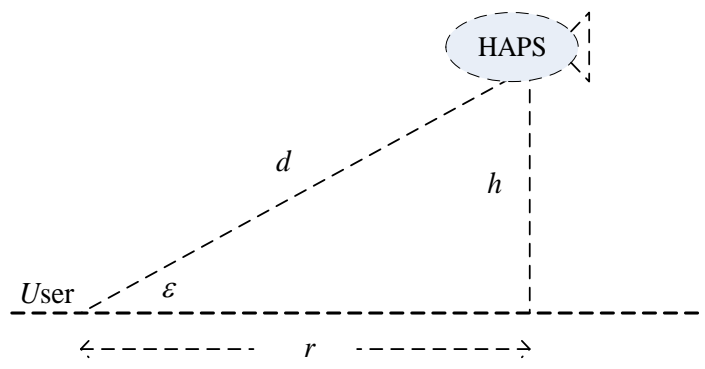

Figure 2. The geometric relationships of a communications system

Within the scope of the near-earth space using stable communications platform as a microwave relay station. With the ground control equipment,entrance equipment and a variety of wireless communication system in the composition of the user.High altitude platform can comprehensive network and satellite ground, also can separate and ground network, as can be seen in Fig.3.The communication platform to keep in sync with the earth's rotation,can reside the air for a long time. High altitude platform communication of good waves transmission characteristics [21-30].Through the platform to realize the ground between the user, platform or platform and satellite communication between the connection. With flexible layout, wide application, the advantages of low cost, safe and reliable.

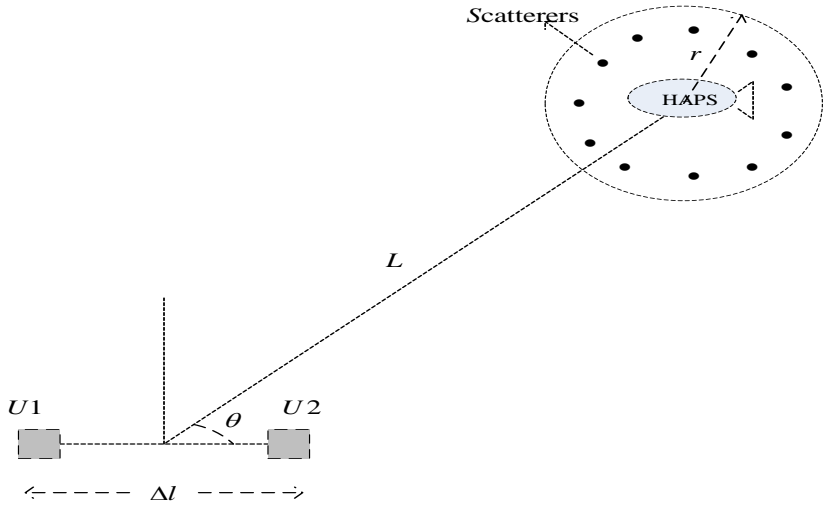

Figure 3. The scatterers of HAPS

\section{CHANNEL MODELING}

The model of channel tap delay in HAPS wireless communication network can be expressed as:

$$
h(t, \tau)=c_{0} \cdot \delta\left(t-t_{0}\right)+\sum_{n=1}^{N} c_{n}(t) \delta\left(t-\tau_{n}\right)
$$

Where, $c_{0}$ is the part of LOS (Line of Sight), $c_{n}(t)$ shows the variable coefficient, which follow the Gaussian distribution. $\tau_{n}$ is the delay time of $\mathrm{n}$ tap.

The envelope of the signal is plural, which can be shown in:

$$
S(t) e^{j \theta(t)}=u(t) e^{j \alpha(t)}+v(t) e^{j \beta(t)}
$$

In the above formula, ${ }^{u(t)}$ is a random variables which obey Rayleigh distribution.

$\alpha(t)$ obey $(0,2 \pi)$ Uniform distribution. $v(t)$ and $\beta(t)$ are the deterministic signals.

The PDF (Probability Density Function) of $S(t)$ be expressed as:

$$
f(s)=\frac{s}{\sigma^{2}} e^{\left(-\frac{s^{2}+v^{2}}{2 \sigma^{2}}\right)} \mathrm{I}_{0}\left(\frac{s v}{\sigma^{2}}\right)
$$

In the above formula, $s>0, \mathrm{I}_{0}$ is the Besell function. The root mean square can be calculated by using this formula:

$$
E\left[s^{2}\right]=v^{2}+2 \sigma^{2}
$$

Where, $v$ is the signal envelope which can receive the direct signal. $2 \sigma^{2}$ is the average power of the HAPS space path.The properties of the Rice distribution is related to the $k$ factor.

$$
k=\frac{v^{2}}{2 \sigma^{2}}
$$

$k$ is the average power. Besell function can be approximated by:

$$
\mathrm{I}_{0}(x)=\frac{e^{x}}{\sqrt{2 \pi x}}, x \rightarrow \infty
$$

Then, $f(s)$ can be expressed as:

$$
f(s)=\sqrt{\frac{s}{2 \pi x}} e^{\left(-\frac{(s-v)^{2}}{2 \sigma^{2}}\right)}
$$

\section{SIMULATION ANALYSIS}

The PDF (Probability Density Function) under different $v$ is shown in Fig.4. 


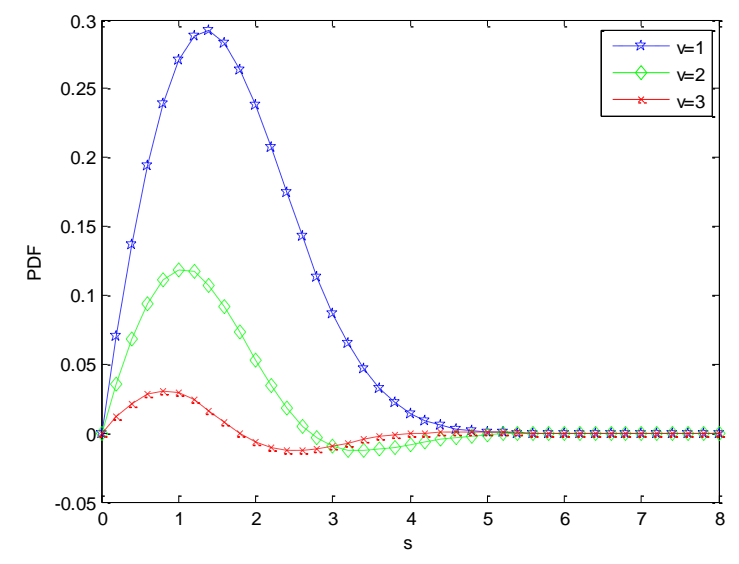

Figure 4. PDF of different $v$

As can be seen from Fig.4.With the increase of $v$, the Rice factor $k$ also increases. Different $k$ values represent different LOS (line-of-sight) transmission conditions. There is always a direct path between users and HAPS.

The distance is set up with $15 \mathrm{~km}$. Relative received signal level under different spacing $\lambda$ is shown in Fig.5.

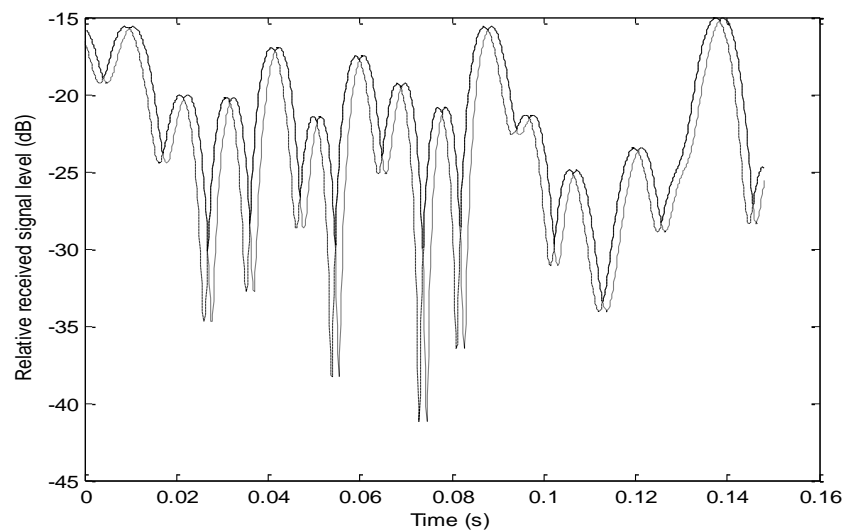

(a) Spacing $1 / 9 \lambda$

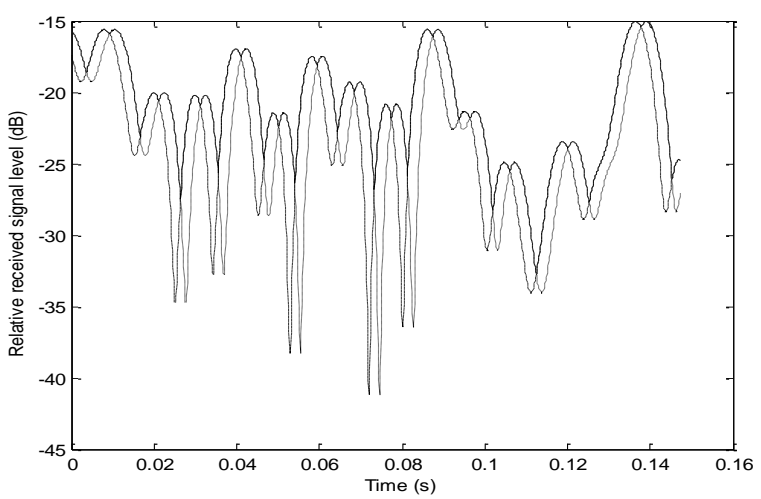

(b) Spacing $1 / 6 \lambda$

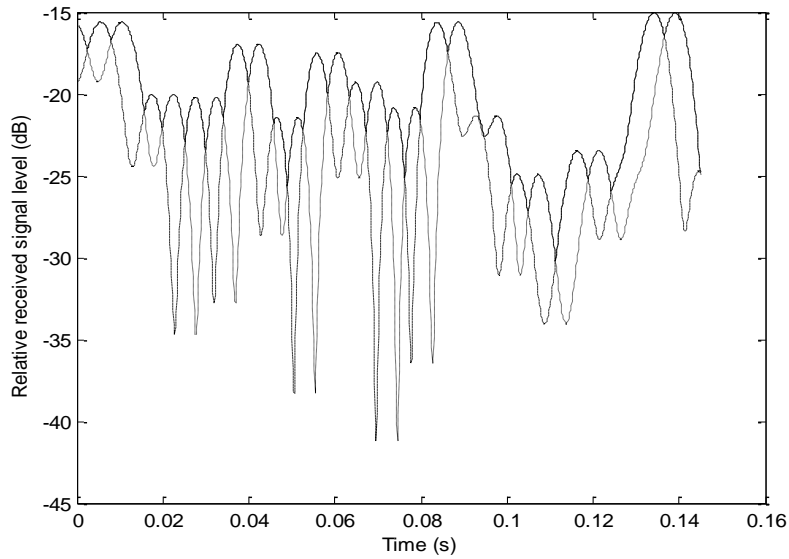

(c) Spacing $1 / 3 \lambda$

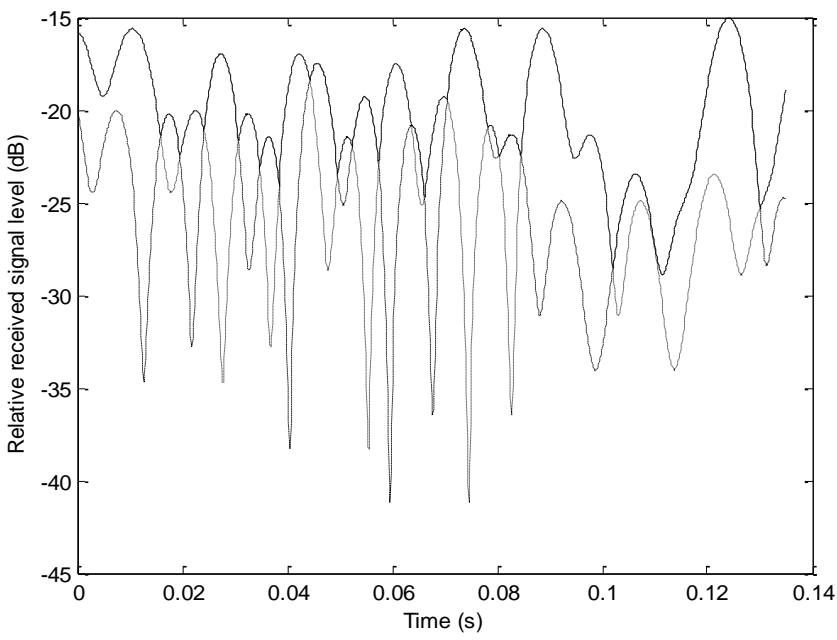

(d) Spacing $\lambda$

Figure 5. Relative received signal level under different spacing $\lambda$

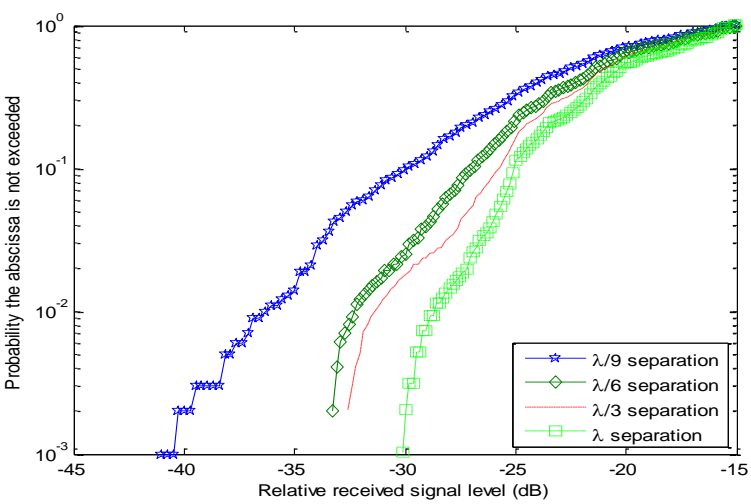

Figure 6. Signal level(dB)

As can be seen from Fig.6,Signal level is different when the spacing distance is different. The smaller the interval, the better of the signal level. 


\section{CONCLUSIONS}

Through the analysis of the wireless communication network of near space HAPS, we can get some conclusions. First of all, relative received signal level is related to the spacing distance. Second, the authentication process for HAPS mobile communication is to confirm the identity of Mobile Station, which is by exchanging information between Mobile Station and Base Station. The next step in our research is to focus on the performance and characteristics of HAPS wireless communication network system which will be measured with an extensive system of sensors, in order to optimize it further.

\section{ACKNOWLEDGMENTS}

The paper are supported by Chongqing Postdoctoral Researchers Research Project Special Funded (Xm2015029), Scientific and Technological Research Program of Chongqing Municipal Education Commission (Grant No.KJ1500926,KJ1600923,KJ1500904,KJ1500920),Chong qing social sciences planning doctoral project (2015BS059), Chongqing Science and Technology Committee Fund of Foundation and Cutting-edge Research Plan(cstc2014jcyjA40007),Natural Science Foundation of China $(61571069,61501065,61502064,61503052,91438104$, 11547148), Ministry of Education of humanities and social sciences research project (16YJC860010).

\section{REFERENCES}

[1] Junil Choi,David J. Love,T.Patrick Bidigare,"Coded distributed diversity:A novel distributed reception technique for wireless communication systems,"IEEE Transactions on Signal Processing, vol.63, no.5,pp.1310-1321(2015)

[2] Jose Acuna; Inigo Cuinas, "Average spectral efficiency of multimodulation cellular systems," IET Communications , vol.7, no.2,pp.146 - 151(2013)

[3] Xiaoyang Liu,Chao Liu,Wanping Liu,"Wind Shear Target Echo Modeling and Simulation,'Discrete Dynamic in Nature and Society, vol.2015,no.4,pp.1-6(2015)

[4] Wen-Qin Wang,Dingde Jiang,"Integrated wireless sensor systems via near-space and satellite platforms:A review,"IEEE Sensors Journal, vol.14,no.11,pp.3903-3914(2014)

[5] Ruimin Zhang,Lu Dong,Changyin Sun, “Adaptive nonsingular terminal sliding mode control design for near space hypersonic vehicles,"'IEEE/CAA Journal of Automatica Sinica,vol.1,no.2,pp.155161(2014)

[6] Guangqiang Chen,Bingyan Chen,Pengfei Li, Peng Bai, Chunqun $\mathrm{Ji}$,"Study of aerodynamic configuration design and wind tunnel test for solar powered buoyancy-lifting vehicle in the nearspace,"Procedia Engineering,vol.99,pp.67-72(2015)

[7] Guanhua Wang,Shanfeng Zhang,Kaishun Wu,et al,"TiM: finegrained rate adaptation in wLANs," IEEE Transactions on Mobile Computing,vol.15,no.3,pp. 748-761(2015)

[8] Jie Hu,Lie-Liang Yang,Lajos Hanzo,"Distributed Multistage cooperative-social-multicast-aided content dissemination in random mobile networks,"IEEE Transactions on Vehicular Technology, vol.64, no.7,pp.3075 - 3089(2015)

[9] Neyre Tekbiyik,Tolga Girici,Elif Uysal-Biyikoglu,Kemal Leblebicioglu, " Proportional fair resource allocation on an energy harvesting downlink,"IEEE Transactions on Wireless Communications, vol.12, no.4,pp.1699-1711(2013)
[10] Jonathan Fink,Alejandro Ribeiro, Vijay Kumar, "Robust control of mobility and communications in autonomous robot teams,"IEEE Access,vol.1,pp.290 - 309(2013)

[11] Jinyuan Su,"Near space as a sui generis zone: A tri-layer approach of delimitation,"Space Policy,vol.29,no.2,pp.90-92(2013)

[12] Bin Jiang,Dezhi Xu,Peng Shi,Cheng Chew Lim,“Adaptive neural observer-based back stepping fault tolerant control for near space vehicle under control effect or damage,"IET Control Theory \& Applications,vol.8,no.9,pp.658-666(2014)

[13] Nouha Baccour,Anis Koubaa,Habib Youssef,Mario Alves,"Reliable link quality estimation in low-power wireless networks and its impact on tree-routing,"Ad Hoc Networks, vol.27,pp.1-25(2015)

[14] Arash Anzalchi,Masood Moghaddami,Amir Moghaddasi,Arif I. Sarwat,Akshay K. Rathore, "A new topology of higher order power filter for single-phase grid-tied voltage-source inverters,"IEEE Transactions on Power Electronics, vol.63,no.12,pp.7511-7522(2016)

[15] Juan J. Gálvez, Pedro M. Ruiz,“Joint link rate allocation, routing and channel assignment in multi-rate multi-channel wireless networks,"Ad Hoc Networks, vol.29,pp.78-98,2015.

[16] Ajinkya Rajandekar;Biplab Sikdar,"A survey of MAC layer issues and protocols for machine-to-machine communications,"IEEE Internet of Things Journal,vol.2, no.2,pp.175 - 186(2015)

[17] Rodolfo Feick,Mauricio Rodríguez,Luciano Ahumada,et al,"Achievable gains of directional antennas in outdoor-indoor propagation environments," IEEE Transactions on Wireless Communications,vol.14, no.3,pp.1447-1456(2015)

[18] Guang Mingxiang,Guo Qing,Gu Xuemai,"Performace evaluation of coverage and wireless link characterstic for HAPS communication,"Chinese Journal of radio science,vol.27,no.4,pp.832839(2012)

[19] Gu Wenzhe,Sun Qibo,Zhang Hai,Yang Fangchun,"A multiple metrics aware routing algorithm for HAPS networks,"Journal of Beijing University of Posts and Telecommunications,vol.35,no.3,pp.5255(2012)

[20] Guan Mingxiang,Guo Qing,Gu Xuemai,"Model and evaluation for performance effects by instability of HAP for HAPS communication,"ACTA Electronica Sinica,vol.40,no.10,pp.19481953(2012)

[21] Wang Xiang,Zhao Shanghong,Zheng Guangwei,"Performance analysis of high altitude platform optical communication links with spatial diversity,"ACTA Optica Sinica,vol.34,no.1,pp.1-7(2014)

[22] Liu xiaoyang,Li yong,Cheng yufeng,"The analysis of the side-lobe clutter in the pulse Doppler radar,"Journal of Computational Information Systems, vol.8,no.4,pp.1671-1677(2012)

[23] Ying Dai,Jie Wu,Chunsheng Xin,"Efficient virtual backbone construction without a common control channel in cognitive radio networks,"IEEE Transactions on Parallel and Distributed Systems,vol.25, no.12, pp.146 - 151,pp.3156-3166(2014)

[24] Israat Tanzeena Haque,Chadi Assi,"Profiling-based indoor localization schemes,"IEEE Systems Journal, vol.9, no.1,pp.7685(2015)

[25] Quoc-Tuan Vien,Brian G. Stewart,Huaglory Tianfield,Huan X. Nguyen,"Efficient cooperative spectrum sensing for three-hop cognitive wireless relay networks,"IET Communications , vol.7, no.2,pp.119-127(2013)

[26] Mona Aggarwal,Parul Garg,Parul Puri,"Dual-hop optical wireless relaying over turbulence channels with pointing error impairments," Journal of Lightwave Technology,vol.32, no. 9,pp.1821-1828(2014)

[27] Mohammad Mansoori,Mehdi Mahdavi,Hamidreza Amini Khorasgani,"Performance analysis of large multi-interface wireless mesh networks with multi-different bandwidth channel,"IEEE Transactions on Mobile Computing, vol.15, no.5, pp.1237-1248(2016)

[28] Xi Zhang,Jun-Hong Cui,Santanu Das,et al,"Underwater wireless communications and networks:theory and application,"IEEE Communications Magazine,vol.53, no.11,pp.40-41(2015) 
[29] Dan Zhang,Luciano Leonel Mendes,Maximilian Matthé,et al,"Expectation propagation for near-optimum detection of MIMOGFDM signals,"IEEE Transactions on Wireless Communications ,vol.15, no.2,pp.1045-1062(2016)
[30] Long Zhao,Xiaodong Wang,Kan Zheng,"Downlink hybrid information and energy transfer with massive MIMO,"IEEE Transactions on Wireless Communications, vol.15, no.2,pp.1309 1322(2016) 